\title{
Tetraspanin Scaffold Proteins Function as Key Regulators of Hematopoietic Stem Cells
}

\author{
Victoria D. Balise ${ }^{1 \dagger}$, Chelsea A. Saito-Reis ${ }^{1 \dagger}$ and Jennifer M. Gillette ${ }^{1,2 *}$ \\ ${ }^{1}$ Department of Pathology, University of New Mexico Health Sciences Center, Albuquerque, NM, United States, \\ ${ }^{2}$ Comprehensive Cancer Center, The University of New Mexico, Albuquerque, NM, United States
}

\section{OPEN ACCESS}

Edited by:

Shinobu Matsuura,

Boston University, United States

Reviewed by:

Laura Schuettpelz,

Washington University in St. Louis,

United States

Owen Tamplin

University of Wisconsin-Madison,

United States

Carlos Cabañas,

Consejo Superior de Investigaciones

Cientificas (CSIC), Spain

*Correspondence:

Jennifer M. Gillette

JGillette@salud.unm.edu

${ }^{t}$ These authors have contributed equally to this work

Specialty section:

This article was submitted to Stem Cell Research,

a section of the journal

Frontiers in Cell and Developmental

Biology

Received: 23 April 2020

Accepted: 19 June 2020

Published: 09 July 2020

Citation:

Balise VD, Saito-Reis CA and Gillette JM (2020) Tetraspanin Scaffold Proteins Function as Key Regulators of Hematopoietic Stem Cells. Front. Cell Dev. Biol. 8:598.

doi: 10.3389/fcell.2020.00598
Hematopoietic stem and progenitor cells (HSPCs) are responsible for the development, maintenance, and regeneration of all the blood forming cells in the body, and as such, are critical for a number of patient therapies. For successful HSPC transplantation, stem cells must traffic through the blood and home to the bone marrow (BM) microenvironment or "niche," which is composed of soluble factors, matrix proteins, and supportive cells. HSPC adhesion to, and signaling with, cellular and extracellular components of the niche provide instructional cues to balance stem cell selfrenewal and differentiation. In this review, we will explore the regulation of these stem cell properties with a focus on the tetraspanin family of membrane proteins. Tetraspanins are molecular scaffolds that uniquely function to distribute proteins into highly organized microdomains comprising adhesion, signaling, and adaptor proteins. As such, tetraspanins contribute to many aspects of cell physiology as mediators of cell adhesion, trafficking, and signaling. We will summarize the many reports that identify tetraspanins as markers of specific HSPC populations. Moreover, we will discuss the various studies establishing the functional importance of tetraspanins in the regulation of essential HSPC processes including quiescence, migration, and niche adhesion. When taken together, studies outlined in this review suggest that several tetraspanins may serve as potential targets to modulate HSPC interactions with the BM niche, ultimately impacting future HSPC therapies.

Keywords: tetraspanin, hematopoietic stem cell, bone marrow niche, quiescence, adhesion, migration

\section{INTRODUCTION}

Hematopoietic stem and progenitor cells (HSPCs) are responsible for the lifelong production of all mature blood and immune cells. As such, HSPCs are critical not only for maintaining homeostasis of the hematopoietic system, but also for responding to stresses such as infection, chemotherapy, or malignant hematopoiesis. The dynamic regulation of the hematopoietic system is not maintained by HSPCs alone, but rather is coordinated with essential support from the surrounding microenvironment or niche (Dexter et al., 1977; Schofield, 1978). HSPCs primarily reside in the bone marrow (BM), which is a complex microenvironment consisting of various cellular and extracellular components all with the capacity to regulate HSPC function and maintenance (Zhang et al., 2003; Morrison and Scadden, 2014). More specifically, there is dynamic interplay between the physical interactions of HSPCs and niche compartments that respond to physiological cues. For example, both circadian oscillations and stress impact the release and trafficking of HSPCs from the BM (Mendez-Ferrer et al., 2009), which leads to their circulation and 
when necessary activation (Huang et al., 2007), or their return to the BM in the process of homing (Lapidot et al., 2005). Clinically, the natural egress and homing of HSPCs are harnessed to collect and successfully transplant HSPCs for treatment of diseases such as hematologic malignancies and BM failure (Hatzimichael and Tuthill, 2010).

Emerging experimental evidence highlights the impact of tetraspanins on the regulation of HSPC activation, HSPC/niche interactions, and the dynamic trafficking of HSPCs into and out of the BM. Thus, in this mini-review, we feature the contributions of specific tetraspanin family members that have been identified as critical modulators of HSPC function.

\section{TETRASPANINS}

The tetraspanin family of membrane scaffold proteins are expressed in all multicellular eukaryotes, with 33 known tetraspanins in humans (Todres et al., 2000; Boucheix and Rubinstein, 2001; Adell et al., 2004). Tetraspanins are characterized by four transmembrane domains, two extracellular loops: one small extracellular loop (SEL or EC1) and one larger extracellular loop (LEL or EC2), a short intracellular loop, and two short intracellular tails (Stipp et al., 2003; Hemler, 2005) (Figure 1A). Tetraspanins are defined by conserved amino acid sequences within EC2 consisting of four or more cysteine residues and a highly conserved CCG motif (Hemler, 2003; Stipp et al., 2003). Additionally, analysis of alternative splice sites within the tetraspanin family recently identified a significant number of novel tetraspanin isoforms with large structural variability (Hochheimer et al., 2019). Tetraspanins protrude only 4-5 $\mathrm{nm}$ above the transmembrane and thus can be overlooked by biochemical and immunological detection (Hemler, 2005). Recent crystal structure studies have provided significant insight including the identification of a cholesterolbinding pocket created by the four transmembrane domains of tetraspanin CD81 (Zimmerman et al., 2016). In fact, cholesterolbinding motifs have now been identified in 30 of the 33 human tetraspanins (Huang et al., 2020). Moreover, the crystal structure for human CD9 describes how its reversed cone-shaped structure generates membrane curvature in a crystalline lipid layer, which also likely explains the localization of CD9 to regions of high membrane curvature (Umeda et al., 2020).

Tetraspanins function as membrane scaffolds through the formation of tetraspanin-enriched microdomains (TEMs), which compartmentalize membrane adhesion and signaling receptors as well as intracellular signaling molecules (Termini and Gillette, 2017; van Deventer et al., 2017). Tetraspanins are also subject to post-translational modifications that include, but are not limited to, palmitoylation and $N$-glycosylation (Termini and Gillette, 2017). For example, tetraspanins CD9, CD37, CD53, CD63, CD81, CD82, and CD151 were found to incorporate palmitate to membrane proximal cysteine residues, which facilitates the stability of tetraspanins within the membrane and promotes formation of TEMs (Charrin et al., 2002; Levy and Shoham, 2005). Additionally, many tetraspanins are glycosylated, which can contribute to their structure and heterogeneity in size (Yunta and Lazo, 2003) and it is also important for regulating proteinprotein interactions (Stipp et al., 2003). While some tetraspanins such as CD9, CD81, and CD82 are ubiquitously expressed (Maecker et al., 1997), other tetraspanins including CD37, CD53, and Tssc6/tspan32 are restricted to hematopoietic cells (Schwartz-Albiez et al., 1988; Maecker et al., 1997). Therefore, the differential expression of tetraspanins in various tissues may translate into specific functions within different cell types. Tetraspanins are also one the most commonly found protein classes in extracellular vesicles (EVs) (Andreu and Yanez-Mo, 2014). In fact, EVs can be categorized based on the enrichment of tetraspanins (Kowal et al., 2016). Collectively, tetraspanins, and the TEMs they assemble, act as molecular facilitators that spatiotemporally organize membrane-associated proteins that drive diverse cellular processes, including those critical for HSPC function, such as quiescence, self-renewal, differentiation, adhesion, migration, and signaling (Table $\mathbf{1}$ and Figure 1B).

\section{TETRASPANINS: HEMATOPOIETIC STEM CELL (HSC) SURFACE MARKERS}

The identification and stratification of HSCs and their progeny is largely based on the use of cell surface markers. For example, long-term hematopoietic stem cells (LT-HSCs), the most primitive of hematopoietic cells with the ability to selfrenew and repopulate the entire blood and immune system, are characterized not by a single surface marker, but rather a combination of surface markers. Several studies have also identified specific tetraspanins as surface markers that can enrich various HSPC populations.

The tetraspanin CD9 was identified as a positive marker for HSCs and HSPCs in both murine and human models. Initially, Bruno et al. discovered high CD9 gene expression within a murine multipotent hematopoietic progenitor cell (HPC) line, but subsequent studies identified CD9 on murine LT-HSC, short-term HSC (ST-HSC), and multipotent progenitor (MPP) cell populations (Bruno et al., 2004; Forsberg et al., 2005). Additionally, $\mathrm{CD} 9^{\text {high }}$ cell populations were identified to not only have HSC phenotypes, but also to be functional in murine transplants (Karlsson et al., 2013). CD9 has also been used as a positive marker to identify human umbilical cord blood stem cells (Zhao et al., 2006). In contrast, a porcine study screening antibodies to identify markers for negative enrichment of HPCs, discovered seven markers, including CD9 (Heinz et al., 2002). Here, HPCs with negative or low expression of CD9 fell within the side population, suggesting that discrepancies may exist between different species with respect to HSPC markers.

Tetraspanin CD82 (KAI1) is also described to be abundantly expressed on the surface of primitive and committed HSPCs isolated from peripheral blood (Burchert et al., 1999). Interestingly, in this study, the level of CD82 expression decreased upon differentiation of $\mathrm{CD}_{4} 4^{+}$HSPCs, but was found to increase in leukemias such as CML, AML, and CLL. More recent proteomic analysis of mouse HSCs and MPPs also identified high CD82 expression in HSCs, when compared to MPPs (Cabezas-Wallscheid et al., 2014). Similarly, gene 
TABLE 1 | Tetraspanins and HSC Regulation.

\begin{tabular}{|c|c|c|c|}
\hline Tetraspanin & Cell type & Function & References \\
\hline \multicolumn{4}{|c|}{ Tetraspanins identified as surface markers } \\
\hline \multirow[t]{5}{*}{ CD9 } & $\begin{array}{l}\text { Murine multipotent hematopoietic progenitor cell } \\
\text { line }\end{array}$ & Marker for HPCs & Bruno et al., 2004 \\
\hline & Murine HSPCs & $\begin{array}{l}\text { Marker on murine LT-HSC, short-term HSC, and multipotent } \\
\text { progenitor cell populations }\end{array}$ & Forsberg et al., 2005 \\
\hline & Murine HSPCs & Marker for murine HSCs & Karlsson et al., 2013 \\
\hline & Human cord blood & Cord blood stem cell maker & Zhao et al., 2006 \\
\hline & Porcine hematopoietic progenitors & Negative enrichment marker for HPCs & Heinz et al., 2002 \\
\hline \multirow[t]{3}{*}{ CD82 } & Human cord blood and human peripheral blood & Identification of tetraspanin on hematopoietic progenitor cells & Burchert et al., 1999 \\
\hline & Murine HSCs and MPP & $\begin{array}{l}\text { Proteomic analysis identified high CD82 expression in HSCs in } \\
\text { comparison to MPP }\end{array}$ & $\begin{array}{l}\text { Cabezas-Wallscheid et al., } \\
2014\end{array}$ \\
\hline & Murine HSCs and HSPCs & $\begin{array}{l}\text { Gene expression analysis demonstrated high CD } 82 \text { expression in } \\
\text { the LT-HSC compared to ST-HSC and MPP populations }\end{array}$ & Hur et al., 2016 \\
\hline CD53 & Murine hematopoietic cell lineages & Differential expression of CD53 within the HSPC population & Guo et al., 2013 \\
\hline CD81 & Human $\mathrm{CD} 4^{+}$cord blood HSPCs & $\begin{array}{l}\text { Marker for differentiation of lymphohematopoietic stem and } \\
\text { progenitor cells }\end{array}$ & Ma et al., 2001 \\
\hline \multicolumn{4}{|c|}{ Tetraspanins identified to regulate quiescence } \\
\hline CD81 & Murine HSCs & Regulation of re-entry of HSC quiescence & Lin et al., 2011 \\
\hline \multirow[t]{3}{*}{ CD82 } & Human peripheral blood HSPCs & $\begin{array}{l}\text { Mediation of HSPC interaction with osteoblasts and HSPC } \\
\text { quiescence }\end{array}$ & Larochelle et al., 2012 \\
\hline & Murine HSCs and HSPCs & Regulation of LT-HSC quiescence & Hur et al., 2016 \\
\hline & Murine HSPCs & Regulation of LT-HSC quiescence & Saito-Reis et al., 2018 \\
\hline \multicolumn{4}{|c|}{ Tetraspanins identified to regulate asymmetric cell division and differentiation } \\
\hline CD63 & $\begin{array}{l}\text { Human umbilical cord blood, bone marrow, and } \\
\text { peripheral blood }\end{array}$ & Marker for asymmetric HSC division & $\begin{array}{l}\text { Beckmann et al., 2007; Giebel } \\
\text { and Beckmann, } 2007\end{array}$ \\
\hline \multirow[t]{3}{*}{ CD53 } & $\begin{array}{l}\text { Human umbilical cord blood, bone marrow, and } \\
\text { peripheral blood }\end{array}$ & Marker for asymmetric HSC division & $\begin{array}{l}\text { Beckmann et al., 2007; Giebel } \\
\text { and Beckmann, } 2007\end{array}$ \\
\hline & Hematopoietic cell line, BaF/3 & B-cell development & Mansson et al., 2007 \\
\hline & Murine bone marrow & Early B-Cell development by regulating IL-7R & Greenberg et al., 2020 \\
\hline \multirow[t]{2}{*}{ CD81 } & Human thymocytes & B lymphocyte development & Tedder et al., 1994 \\
\hline & Human blood samples & Decreased memory B cell numbers in CD81-deficient patients & van Zelm et al., 2010 \\
\hline \multirow[t]{4}{*}{ CD9 } & Human bone marrow CD34+ & Stimulates differentiation to megakaryocytic lineages & Clay et al., 2001 \\
\hline & Human cord blood CD34+ & Dendritic cell marker & Caux et al., 1996 \\
\hline & Murine megakaryocyte-erythroid progenitors & High expression of CD9 promotes self- renewal & Weiss-Gayet et al., 2016 \\
\hline & Pluripotent hematopoietic cell line, EML-C1 & $\begin{array}{l}\text { Regulation of HSC differentiation, proliferation, and self-renewal } \\
\text { through stromal cell expression }\end{array}$ & $\begin{array}{l}\text { Aoyama et al., 1999; Oritani } \\
\text { et al., } 2000\end{array}$ \\
\hline
\end{tabular}

$\overline{H P C}$, hematopoietic progenitor cell; HSPC, hematopoietic stem and progenitor cell; MPP, multipotent progenitor; LT-HSC, long-term hematopoietic stem cell; ST-HSC, short-term hematopoietic stem cell.

expression analysis demonstrated that CD82 was expressed predominantly in the LT-HSC rather than the ST-HSC and MPP populations (Hur et al., 2016). In contrast, this study found that other members of the tetraspanin superfamily (CD9, CD37, CD81, and CD151) were expressed in every HSPC population at the mRNA level. However, Guo et al. (2013) identified CD53 to be differentially expressed within the murine HSPC population by single-cell gene expression analysis. Additionally, CD81 was described as a marker for the development of lymphohematopoietic stem and progenitor cells (Ma et al., 2001) and the expression of Tssc6 has been confirmed on HPCs and various HSC lines (Robb et al., 2001). While tetraspanin expression appears to vary across HSPC populations, it is clear that certain tetraspanins can be used to enrich specific hematopoietic cell fractions, which may improve stem cell therapies.

\section{TETRASPANINS: HSC QUIESCENCE}

Within the BM microenvironment, HSCs primarily reside in a quiescent state (Wilson et al., 2008). However, upon injury, HSCs are activated into cycle, but then must ultimately return to quiescence (Wilson et al., 2008). Therefore, HSC quiescence is not only important for protecting the stem cell pool from mutations accumulated via active cycling, but also for sustaining the HSC pool over the lifespan of an organism (Li, 2011). Tetraspanins CD81 and 


\section{A Tetraspanin Structure B Bone Marrow Niche}
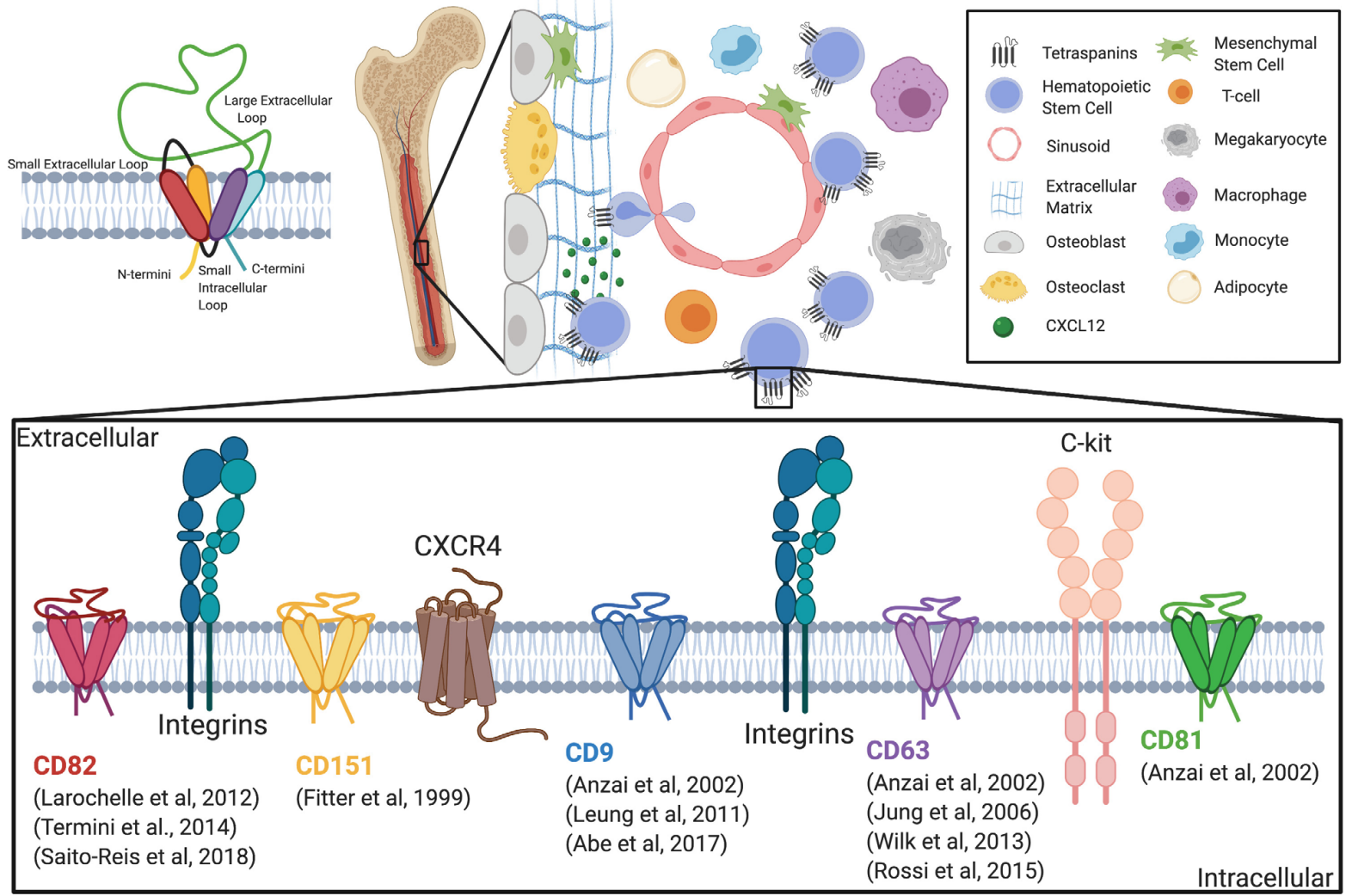

FIGURE 1 | Tetraspanins regulate HSC adhesion, migration, and signaling within the niche. (A) Schematic of the tetraspanin molecular structure. (B) Diagram depicting the bone marrow niche and the cell types that regulate HSC function including mesenchymal stem cells, endothelial cells, megakaryocytes, macrophages, osteoblasts, osteoclasts, and adipocytes. Additionally, a magnified diagram of the HSPC membrane depicts different tetraspanins involved in adhesion, migration, and signaling, as well as known interacting molecules.

CD82 have both been described as important modulators of HSC quiescence.

The spatial distribution of CD81 on the surface of murine HSCs was shown to be important for the re-entry of HSCs into quiescence from a highly proliferative state (Lin et al., 2011). More specifically, the polarization of CD81 was shown to promote the deactivation of Akt signaling and the nuclear translocation of FoxOla, leading to HSC quiescence. Similarly, work from our group identified a change in the distribution of CD82 as human CD34 ${ }^{+}$cells progress through the cell cycle (Larochelle et al., 2012). Quiescent, G0 cells were found to have a polarized organization of CD82, which was redistributed throughout the plasma membrane upon cell cycle entry. Together, these data highlight the critical contribution of tetraspanin membrane organization to the signal transduction driving quiescence. In both studies, the tetraspanin membrane organization was described at the micron scale. Therefore, future studies will be required to determine how tetraspanin assembly into nanoscale TEMs and the dynamic modulation of TEMs impacts quiescence signaling of HSCs.

A number of tetraspanin knock out $(\mathrm{KO})$ mice have been generated, but up until now, only two have displayed an
HSC defect. CD82KO animals exhibit a reduction in LTHSCs, resulting from increased stem cell activation and reduced quiescence signaling (Hur et al., 2016; Saito-Reis et al., 2018). In contrast, HSCs from CD81KO mice appear to proliferate similarly to control animals, but demonstrate a reduced ability to reenter quiescence after stimulation (Lin et al., 2011). Interestingly, the signaling molecules described to be involved in the regulation of quiescence include both Akt and TGF $\beta$ signaling (Lin et al., 2011; Hur et al., 2016). The modulation of Akt and TGF $\beta$ signaling by CD81 and CD82, respectively, likely involves the scaffolding property of these tetraspanins and their ability to cluster receptors and downstream signaling molecules into TEMs. Future experiments utilizing advanced imaging technologies will be required to confirm how tetraspanins and the dynamic assembly of TEMs influence these quiescence signaling pathways.

\section{TETRASPANINS: ASYMMETRIC DIVISION AND DIFFERENTIATION}

The balance between HSC self-renewal and differentiation is thought to be regulated by asymmetric cell division, where a cell 
produces a daughter cell that retains intrinsic stem cell properties plus one that initiates differentiation (Ho, 2005; Caocci et al., 2017). Previous work identified a series of molecules expressed on HSCs that undergo asymmetric division, including CD133, CD71, CD62L, CD34, and the tetraspanins, CD53 and CD63 (Beckmann et al., 2007; Giebel and Beckmann, 2007). In fact, CD53, in combination with CD63 served as more stringent markers for asymmetric division than the previously described CD133 and CD34 expression profiles (Beckmann et al., 2007; Giebel and Beckmann, 2007). In this study, both CD53 and CD63 were linked to the endosomal compartment, which plays a critical role in protein trafficking (Beckmann et al., 2007). Moreover, it has been suggested that the asymmetric segregation of endosomes might provide a more general and evolutionary conserved mechanism for asymmetric cell division (Murke et al., 2015).

Specific tetraspanin expression has also been linked to differentiation of hematopoietic cells. For example, CD53 was shown to be a genetic target for Early B-cell Factor-1, a critical transcription factor for B-cell development (Mansson et al., 2007). More recently, an increase in CD53 expression was demonstrated in early B-cell development where a physical interaction between CD53 and the Interleukin-7 receptor modulates key signaling in early B cell differentiation (Greenberg et al., 2020).

Also critical for B lymphocyte development and humoral immunity is the CD19-CD21-CD81 complex (Tedder et al., 1994), where tetraspanin CD81 associates with CD19 in the endoplasmic reticulum and regulates CD19 surface transport. In fact, CD81-deficient patients were characterized by decreased memory B cell numbers and an absence of CD19 surface expression, which further demonstrates an essential, nonredundant role of CD81 (van Zelm et al., 2010). Biochemical analysis of the CD19-CD21-CD81 complex also identified an association with tetraspanins CD82 and CD9 (Horvath et al., 1998). A comprehensive description of tetraspanin activity in $B$ cell development can be found in the following review (Zou et al., 2018).

Tetraspanin CD9 is also a regulator of HSPC differentiation with specific roles in the differentiation of the megakaryocytic, B-lymphoid, and myeloid lineages (Brosseau et al., 2018). For instance, high CD9 expression on $\mathrm{CD}_{3} 4^{+}$cells promotes differentiation to megakaryocytic lineages (Clay et al., 2001). Additionally, CD9 surface expression was identified to characterize a population of dendritic cells that were differentiated in vitro from CD $34^{+}$HPCs isolated from human cord blood (Caux et al., 1996). Moreover, a subset of CD9High committed megakaryocytic progenitors were shown to exhibit self-renewal and lineage plasticity downstream of Notch stimulation (Weiss-Gayet et al., 2016). Further study of CD9 revealed its expression on a large variety of other hematopoietic cells, including platelets, T lymphocytes, mast cells, eosinophils, and basophils (Fernvik et al., 1995; Tai et al., 1996) as well as critical BM stromal cells that can also regulate HSPC activity (Aoyama et al., 1999; Oritani et al., 2000). For example, when the pluripotent hematopoietic cell line, EML-C1, was cultured on stromal cells treated with an anti-CD9 antibody, a block in HSC differentiation, proliferation, and self-renewal was measured
(Aoyama et al., 1999). Interestingly, CD9 and CD63 were also identified to be enriched in EVs produced by stromal cell lines that can differentially support HSPCs (Stik et al., 2017). It has been suggested that tetraspanins within EVs may facilitate target cell binding and uptake (Morelli et al., 2004); however, whether tetraspanins serve this role in HSC/niche communication requires further study. At this time, it is clear that tetraspanins play a key role in the regulation of niche interactions, which will be further discussed below.

\section{TETRASPANINS: HSC ADHESION, MIGRATION, AND SIGNALING WITHIN THE NICHE}

The BM niche is a particularly complex microenvironment that consists of a diverse cellular repertoire critical for regulating HSC function and maintenance. Currently, it is believed that HSCs are predominantly localized to the perivascular region of the BM, with endothelial cells and mesenchymal stromal cells serving to secrete factors that promote HSC maintenance (Crane et al., 2017). However, other cell types also directly or indirectly regulate HSC niches, including megakaryocytes, macrophages, osteoblasts, osteoclasts, adipocytes, and other stromal cell populations (Morrison and Scadden, 2014). Additionally, the extracellular matrix (ECM) within the BM acts as a supportive tissue for the maintenance of HSCs (Discher et al., 2009). The different components of the BM microenvironment are depicted in Figure 1B and have been extensively reviewed (Crane et al., 2017; Szade et al., 2018; Pinho and Frenette, 2019).

The dynamic adhesion and migration behaviors of HSCs within the BM are significantly regulated by multiple adhesion proteins, including the integrin family of adhesion molecules (Klamer and Voermans, 2014). Integrin complexes, $\alpha 4 \beta 1$ and $\alpha L \beta 2$ play an important role in HSC adhesion to the vasculature to aide in trans-endothelial migration (Peled et al., 2000). Additionally, integrin complexes $\alpha 4 \beta 1$ and $\alpha 6 \beta 1$, are both important for homing of HSCs to the BM. Studies using antibodies to block either $\alpha 4$ or $\alpha 6$ resulted in an inhibition of BM homing of HSPCs (Papayannopoulou et al., 1995; Qian et al., 2006). Moreover, in combination, $\alpha 4$ and $\alpha 6$ antibodies synergistically inhibited homing of STHSCs (Qian et al., 2006). Interestingly, an increase in homing of progenitors to spleen was only detected with antibodies against $\alpha 4$ (Papayannopoulou et al., 1995), suggesting distinct roles of integrins in HSCs homing. Both $\alpha 4 \beta 1$ and $\alpha 5 \beta 1$ were also found to mediate chemotaxis of $\mathrm{CD} 34^{+} \mathrm{HPCs}$ on fibronectin, while $\alpha 4 \beta 1$ alone mediates adhesion (Carstanjen et al., 2005). Integrin interactions with tetraspanins have been well characterized (Berditchevski, 2001). In fact, tetraspanins have been described to impact integrin expression, signaling, and compartmentalization. Thus, much of the contribution of tetraspanins to the regulation of HSC adhesion and migration within the niche involves integrin-mediated interactions (Figure 1B).

Our work identified a critical role for the tetraspanin CD82 in mediating HSPC/niche adhesion. CD82 was found to be enriched 
at the site of HSPC contact with osteoblasts and pre-treatment of $\mathrm{CD} 34^{+}$cells with an anti-CD82 monoclonal antibody resulted in reduced adhesion, homing, and engraftment (Larochelle et al., 2012). Follow-up studies with a human hematopoietic cell line demonstrated that CD82 promotes fibronectin adhesion through the regulation of integrin $\alpha 4 \beta 1$ organization (Termini et al., 2014). Super-resolution imaging studies identified CD82 as a modulator of integrin density, which contributed to changes in ECM adhesion. Similarly, a previous study focused on CD151 identified interactions with integrins $\beta 1$ and $\alpha \operatorname{IIb} \beta 3$ as critical for promoting HSPC adhesion to various ECMs (Fitter et al., 1999) and both CD81 and CD82 were shown to mediate $\alpha 4 \beta 1$ adhesion of erythroblasts to Vascular Cell Adhesion molecule-1 (Spring et al., 2013). More recently, our work using the CD82KO mouse model identified a homing and engraftment defect of HSPCs, which was linked to altered Rac1 activity (Saito-Reis et al., 2018), further illustrating that CD82 may serve as a therapeutic target to modulate HSPC adhesion and migration.

Tetraspanin CD9 has also been described as a regulator of HSPC adhesion and homing. Pretreatment of cord blood $\mathrm{CD}^{+} 4^{+}$cells with an anti-CD9 monoclonal antibody inhibited CXCL12-mediated transendothelial migration; however, adhesion to fibronectin and endothelial cells was enhanced (Leung et al., 2011). Additionally, antibody pretreatment of $\mathrm{CD}^{+} 4^{+}$cells significantly impaired their homing to the $\mathrm{BM}$ and sorted $\mathrm{CD}^{+} 4^{+} \mathrm{CD}^{-}$cells displayed lower BM homing capacity compared with that of total $\mathrm{CD}^{+}{ }^{+}$cells. More recently, a separate group characterized human $\mathrm{CD}^{-} 4^{-}$HSCs isolated from cord blood, finding that engraftment in mice and sheep was limited due to a decrease in CD9 expression and an increase in the inhibitory homing molecule, CD26 (Abe et al., 2017). Collectively, these data demonstrate that CD9 expression also contributes to HSC migration and niche adhesion, although identification of its mechanistic role and interacting partners remains unclear. Studies evaluating mature hematopoietic cells have identified a role for CD9 in regulating cell adhesionmediated by integrins, $\alpha \mathrm{L} \beta 2$ and $\alpha 5 \beta 1$ (Reyes et al., 2015, 2018; Machado-Pineda et al., 2018). Thus, future studies focused on the role of CD9 and HSCs are likely to uncover key integrin interactions.

In addition to its enrichment in HSC endosomes, tetraspanin CD63 was found to interact with the tissue inhibitor of metalloproteinase-1 (TIMP1), a protein important for HSC quiescence and long-term engraftment (Jung et al., 2006). TIMP1 was shown to bind to the CD63/ $\beta 1$ integrin complex on the surface of human $\mathrm{CD}^{+} 4^{+}$HSPCs to induce adhesion and migration (Wilk et al., 2013). Moreover, this group determined that homing and short-term engraftment of HSPCs were also increased upon exogenous stimulation with TIMP1. The interaction of TIMP1 and CD63 was also shown to impact HSPC proliferation through the activation of the PI3K/AKT signaling pathway (Rossi et al., 2015) and the enrichment of myeloid progenitors, impacting granulopoiesis (Kobuch et al., 2015). Additionally, HSC proliferation, self-renewal, and maintenance of niche interactions are regulated by the tyrosine kinase receptor c-kit (CD117) expressed on the surface of HSPCs and its ligand stem cell factor (Barker, 1997; Kent et al., 2008). Using a combination of immunoprecipitation and co-localization experiments, tetraspanins CD9, CD63, and CD81 were shown to interact with c-kit (Anzai et al., 2002). Functionally, this study suggested that tetraspanins negatively modulate c-kit signaling and thus may regulate receptor sensitivity to ligand within the BM niche.

\section{CONCLUSION}

Tetraspanins are a family of proteins that regulate multiple cellular processes through their organization of membraneassociated proteins into TEMs. This review explores the tetraspanins currently known to modulate various HSPC functions. Our description of the diverse HSPC processes regulated by several tetraspanins highlights the need to further investigate the mechanistic role for tetraspanins in the regulation of HSC signaling and niche interactions. Future studies focused on how tetraspanins dynamically modulate the compartmentalization of critical HSPC signaling and adhesion molecules will help us understand the specific mechanisms used by this family of proteins to control unique HSPC activities. Now that sophisticated imaging techniques are becoming more widely available, we are likely to learn more about how tetraspanins contribute to the formation and stabilization of signaling and adhesion complexes essential for HSC function. As several tetraspanin family members are enriched in HSPCs, it will also be critical to investigate how tetraspanins may work in concert to modulate interacting protein partners and downstream signaling that contribute to HSPC/niche interactions. Finally, the generation and analysis of HSC-specific tetraspanin $\mathrm{KO}$ mice will be essential for separating the critical roles for tetraspanins specifically within HSCs from those required in the BM niche. Collectively, an advanced understanding of how tetraspanins contribute to HSPC function may lead to future breakthroughs in the isolation and the therapeutic use of HSPCs.

\section{AUTHOR CONTRIBUTIONS}

VB, CS-R, and JG contributed to manuscript writing. VB generated the figures. All authors approved the final version of the manuscript.

\section{FUNDING}

This work was supported by the following grants: NIH R01 HL12248301 (to JG), NIH K12 GM088021 (to AWN for VB), NIH T32 HL007736 (to TCR for CS-R), and American Cancer Society Research Scholar Grant \#130675 (to JG).

\section{ACKNOWLEDGMENTS}

The authors thank M. Floren and E. M. Pascetti for critical review of the manuscript. Figures were created with BioRender.com. 


\section{REFERENCES}

Abe, T., Matsuoka, Y., Nagao, Y., Sonoda, Y., and Hanazono, Y. (2017). CD34negative hematopoietic stem cells show distinct expression profiles of homing molecules that limit engraftment in mice and sheep. Int. J. Hematol. 106, 631-637. doi: 10.1007/s12185-017-2290-5

Adell, T., Gamulin, V., Perovic-Ottstadt, S., Wiens, M., Korzhev, M., Muller, I. M., et al. (2004). Evolution of metazoan cell junction proteins: the scaffold protein MAGI and the transmembrane receptor tetraspanin in the demosponge Suberites domuncula. J. Mol. Evol. 59, 41-50. doi: 10.1007/s00239-004-2602-2

Andreu, Z., and Yanez-Mo, M. (2014). Tetraspanins in extracellular vesicle formation and function. Front. Immunol. 5:442. doi: 10.3389/fimmu.2014. 00442

Anzai, N., Lee, Y., Youn, B. S., Fukuda, S., Kim, Y. J., Mantel, C., et al. (2002). C-kit associated with the transmembrane 4 superfamily proteins constitutes a functionally distinct subunit in human hematopoietic progenitors. Blood 99, 4413-4421. doi: 10.1182/blood.v99.12.4413

Aoyama, K., Oritani, K., Yokota, T., Ishikawa, J., Nishiura, T., Miyake, K., et al. (1999). Stromal cell CD9 regulates differentiation of hematopoietic stem/progenitor cells. Blood 93, 2586-2594. doi: 10.1182/blood.v93.8.2586. 408k23_2586_2594

Barker, J. E. (1997). Early transplantation to a normal microenvironment prevents the development of Steel hematopoietic stem cell defects. Exp. Hematol. 25, 542-547.

Beckmann, J., Scheitza, S., Wernet, P., Fischer, J. C., and Giebel, B. (2007). Asymmetric cell division within the human hematopoietic stem and progenitor cell compartment: identification of asymmetrically segregating proteins. Blood 109, 5494-5501. doi: 10.1182/blood-2006-11-055921

Berditchevski, F. (2001). Complexes of tetraspanins with integrins: more than meets the eye. J. Cell Sci. 114(Pt 23), 4143-4151.

Boucheix, C., and Rubinstein, E. (2001). Tetraspanins. Cell Mol. Life Sci. 58, 1189-1205. doi: 10.1007/PL00000933

Brosseau, C., Colas, L., Magnan, A., and Brouard, S. (2018). CD9 tetraspanin: a new pathway for the regulation of inflammation? Front. Immunol. 9:2316. doi: 10.3389/fimmu.2018.02316

Bruno, L., Hoffmann, R., McBlane, F., Brown, J., Gupta, R., Joshi, C., et al. (2004). Molecular signatures of self-renewal, differentiation, and lineage choice in multipotential hemopoietic progenitor cells in vitro. Mol. Cell Biol. 24, 741-756. doi: $10.1128 / \mathrm{mcb} \cdot 24.2 .741-756.2004$

Burchert, A., Notter, M., Dietrich Menssen, H., Schwartz, S., Knauf, W., Neubauer, A., et al. (1999). CD82 (KAI1), a member of the tetraspan family, is expressed on early haemopoietic progenitor cells and up-regulated in distinct human leukaemias. Br. J. Haematol. 107, 494-504. doi: 10.1046/j.1365-2141.1999. 01741.x

Cabezas-Wallscheid, N., Klimmeck, D., Hansson, J., Lipka, D. B., Reyes, A., Wang, Q., et al. (2014). Identification of regulatory networks in HSCs and their immediate progeny via integrated proteome, transcriptome, and DNA methylome analysis. Cell Stem Cell 15, 507-522. doi: 10.1016/j.stem.2014.07. 005

Caocci, G., Greco, M., and La Nasa, G. (2017). Bone marrow homing and engraftment defects of human hematopoietic stem and progenitor cells. Mediterr. J. Hematol. Infect. Dis. 9:e2017032. doi: 10.4084/MJHID.2017.032

Carstanjen, D., Gross, A., Kosova, N., Fichtner, I., and Salama, A. (2005). The alpha4beta1 and alpha5beta1 integrins mediate engraftment of granulocytecolony-stimulating factor-mobilized human hematopoietic progenitor cells. Transfusion 45, 1192-1200. doi: 10.1111/j.1537-2995.2005.00172.x

Caux, C., Vanbervliet, B., Massacrier, C., Dezutter-Dambuyant, C., de Saint-Vis, B., Jacquet, C., et al. (1996). CD34+ hematopoietic progenitors from human cord blood differentiate along two independent dendritic cell pathways in response to GM-CSF+TNF alpha. J. Exp. Med. 184, 695-706. doi: 10.1084/jem.184.2.695

Charrin, S., Manie, S., Oualid, M., Billard, M., Boucheix, C., and Rubinstein, E. (2002). Differential stability of tetraspanin/tetraspanin interactions: role of palmitoylation. FEBS Lett. 516, 139-144. doi: 10.1016/s0014-5793(02)02522-x

Clay, D., Rubinstein, E., Mishal, Z., Anjo, A., Prenant, M., Jasmin, C., et al. (2001). CD9 and megakaryocyte differentiation. Blood 97, 1982-1989. doi: 10.1182/ blood.v97.7.1982

Crane, G. M., Jeffery, E., and Morrison, S. J. (2017). Adult haematopoietic stem cell niches. Nat. Rev. Immunol. 17, 573-590. doi: 10.1038/nri.2017.53
Dexter, T. M., Allen, T. D., and Lajtha, L. G. (1977). Conditions controlling the proliferation of haemopoietic stem cells in vitro. J. Cell Physiol. 91, 335-344. doi: 10.1002/jcp.1040910303

Discher, D. E., Mooney, D. J., and Zandstra, P. W. (2009). Growth factors, matrices, and forces combine and control stem cells. Science 324, 1673-1677. doi: 10 $1126 /$ science. 1171643

Fernvik, E., Hallden, G., Hed, J., and Lundahl, J. (1995). Intracellular and surface distribution of CD9 in human eosinophils. APMIS 103, 699-706. doi: 10.1111/ j.1699-0463.1995.tb01426.x

Fitter, S., Sincock, P. M., Jolliffe, C. N., and Ashman, L. K. (1999). Transmembrane 4 superfamily protein CD151 (PETA-3) associates with beta 1 and alpha IIb beta 3 integrins in haemopoietic cell lines and modulates cell-cell adhesion. Biochem. J. 338(Pt 1), 61-70. doi: 10.1042/bj3380061

Forsberg, E. C., Prohaska, S. S., Katzman, S., Heffner, G. C., Stuart, J. M., and Weissman, I. L. (2005). Differential expression of novel potential regulators in hematopoietic stem cells. PLoS Genet. 1:e28. doi: 10.1371/journal.pgen.0010028

Giebel, B., and Beckmann, J. (2007). Asymmetric cell divisions of human hematopoietic stem and progenitor cells meet endosomes. Cell Cycle 6, 22012204. doi: $10.4161 /$ cc.6.18.4658

Greenberg, Z. J., Monlish, D. A., Bartnett, R. L., Yang, Y., Shen, G., Li, W., et al. (2020). The tetraspanin CD53 regulates early B cell development by promoting IL-7R signaling. J. Immunol. 204, 58-67. doi: 10.4049/jimmunol.1900539

Guo, G., Luc, S., Marco, E., Lin, T. W., Peng, C., Kerenyi, M. A., et al. (2013). Mapping cellular hierarchy by single-cell analysis of the cell surface repertoire. Cell Stem Cell 13, 492-505. doi: 10.1016/j.stem.2013.07.017

Hatzimichael, E., and Tuthill, M. (2010). Hematopoietic stem cell transplantation. Stem. Cells Clon. 3, 105-117. doi: 10.2147/SCCAA.S6815

Heinz, M., Huang, C. A., Emery, D. W., Giovino, M. A., LeGuern, A., KurillaMahon, B., et al. (2002). Use of CD9 expression to enrich for porcine hematopoietic progenitors. Exp. Hematol. 30, 809-815. doi: 10.1016/s0301$472 \times(02) 00835-4$

Hemler, M. E. (2003). Tetraspanin proteins mediate cellular penetration, invasion, and fusion events and define a novel type of membrane microdomain. Annu. Rev. Cell Dev. Biol. 19, 397-422. doi: 10.1146/annurev.cellbio.19.111301.153609

Hemler, M. E. (2005). Tetraspanin functions and associated microdomains. Nat. Rev. Mol. Cell Biol. 6, 801-811. doi: 10.1038/nrm1736

Ho, A. D. (2005). Kinetics and symmetry of divisions of hematopoietic stem cells. Exp. Hematol. 33, 1-8. doi: 10.1016/j.exphem.2004.09.004

Hochheimer, N., Sies, R., Aschenbrenner, A. C., Schneider, D., and Lang, T. (2019). Classes of non-conventional tetraspanins defined by alternative splicing. Sci. Rep. 9:14075. doi: 10.1038/s41598-019-50267-0

Horvath, G., Serru, V., Clay, D., Billard, M., Boucheix, C., and Rubinstein, E. (1998). CD19 is linked to the integrin-associated tetraspans CD9, CD81, and CD82. J. Biol. Chem. 273, 30537-30543. doi: 10.1074/jbc.273.46.30537

Huang, C., Hays, F. A., Tomasek, J. J., Benyajati, S., and Zhang, X. A. (2020). Tetraspanin CD82 interaction with cholesterol promotes extracellular vesiclemediated release of ezrin to inhibit tumour cell movement. J. Extracell. Ves. 9:1692417. doi: 10.1080/20013078.2019.1692417

Huang, X., Cho, S., and Spangrude, G. J. (2007). Hematopoietic stem cells: generation and self-renewal. Cell Death Differ. 14, 1851-1859. doi: 10.1038/sj. cdd. 4402225

Hur, J., Choi, J. I., Lee, H., Nham, P., Kim, T. W., Chae, C. W., et al. (2016). CD82/KAI1 maintains the dormancy of long-term hematopoietic stem cells through interaction with DARC-expressing macrophages. Cell Stem Cell 18, 508-521. doi: 10.1016/j.stem.2016.01.013

Jung, K. K., Liu, X. W., Chirco, R., Fridman, R., and Kim, H. R. (2006). Identification of CD63 as a tissue inhibitor of metalloproteinase-1 interacting cell surface protein. EMBO J. 25, 3934-3942. doi: 10.1038/sj.emboj.760 1281

Karlsson, G., Rorby, E., Pina, C., Soneji, S., Reckzeh, K., Miharada, K., et al. (2013). The tetraspanin CD9 affords high-purity capture of all murine hematopoietic stem cells. Cell Rep. 4, 642-648. doi: 10.1016/j.celrep.2013.07.020

Kent, D., Copley, M., Benz, C., Dykstra, B., Bowie, M., and Eaves, C. (2008). Regulation of hematopoietic stem cells by the steel factor/KIT signaling pathway. Clin. Cancer Res. 14, 1926-1930. doi: 10.1158/1078-0432.CCR-075134

Klamer, S., and Voermans, C. (2014). The role of novel and known extracellular matrix and adhesion molecules in the homeostatic and regenerative bone 
marrow microenvironment. Cell Adh. Migr. 8, 563-577. doi: 10.4161/19336918. 2014.968501

Kobuch, J., Cui, H., Grunwald, B., Saftig, P., Knolle, P. A., and Kruger, A. (2015). TIMP-1 signaling via CD63 triggers granulopoiesis and neutrophilia in mice. Haematologica 100, 1005-1013. doi: 10.3324/haematol.2014.121590

Kowal, J., Arras, G., Colombo, M., Jouve, M., Morath, J. P., Primdal-Bengtson, B., et al. (2016). Proteomic comparison defines novel markers to characterize heterogeneous populations of extracellular vesicle subtypes. Proc. Natl. Acad. Sci. U.S.A. 113, E968-E977. doi: 10.1073/pnas.1521230113

Lapidot, T., Dar, A., and Kollet, O. (2005). How do stem cells find their way home? Blood 106, 1901-1910. doi: 10.1182/blood-2005-04-1417

Larochelle, A., Gillette, J. M., Desmond, R., Ichwan, B., Cantilena, A., Cerf, A., et al. (2012). Bone marrow homing and engraftment of human hematopoietic stem and progenitor cells is mediated by a polarized membrane domain. Blood 119, 1848-1855. doi: 10.1182/blood-2011-08-371583

Leung, K. T., Chan, K. Y., Ng, P. C., Lau, T. K., Chiu, W. M., Tsang, K. S., et al. (2011). The tetraspanin CD9 regulates migration, adhesion, and homing of human cord blood CD34+ hematopoietic stem and progenitor cells. Blood 117, 1840-1850. doi: 10.1182/blood-2010-04-281329

Levy, S., and Shoham, T. (2005). The tetraspanin web modulates immunesignalling complexes. Nat. Rev. Immunol. 5, 136-148. doi: 10.1038/nri1548

Li, J. (2011). Quiescence regulators for hematopoietic stem cell. Exp. Hematol. 39, 511-520. doi: 10.1016/j.exphem.2011.01.008

Lin, K. K., Rossi, L., Boles, N. C., Hall, B. E., George, T. C., and Goodell, M. A. (2011). CD81 is essential for the re-entry of hematopoietic stem cells to quiescence following stress-induced proliferation via deactivation of the Akt pathway. PLoS Biol. 9:e1001148. doi: 10.1371/journal.pbio.1001148

Ma, F., Wada, M., Yoshino, H., Ebihara, Y., Ishii, T., Manabe, A., et al. (2001). Development of human lymphohematopoietic stem and progenitor cells defined by expression of CD34 and CD81. Blood 97, 3755-3762. doi: 10.1182/ blood.v97.12.3755

Machado-Pineda, Y., Cardenes, B., Reyes, R., Lopez-Martin, S., Toribio, V., Sanchez-Organero, P., et al. (2018). CD9 controls integrin alpha5betalmediated cell adhesion by modulating its association with the metalloproteinase ADAM17. Front. Immunol. 9:2474. doi: 10.3389/fimmu.2018.02474

Maecker, H. T., Todd, S. C., and Levy, S. (1997). The tetraspanin superfamily: molecular facilitators. FASEB J. 11, 428-442. doi: 10.1096/fasebj.11.6.9194523

Mansson, R., Lagergren, A., Hansson, F., Smith, E., and Sigvardsson, M. (2007). The CD53 and CEACAM-1 genes are genetic targets for early B cell factor. Eur. J. Immunol. 37, 1365-1376. doi: 10.1002/eji.200636642

Mendez-Ferrer, S., Chow, A., Merad, M., and Frenette, P. S. (2009). Circadian rhythms influence hematopoietic stem cells. Curr. Opin. Hematol. 16, 235-242. doi: 10.1097/MOH.0b013e32832bd0f5

Morelli, A. E., Larregina, A. T., Shufesky, W. J., Sullivan, M. L., Stolz, D. B., Papworth, G. D., et al. (2004). Endocytosis, intracellular sorting, and processing of exosomes by dendritic cells. Blood 104, 3257-3266. doi: 10.1182/blood-200403-0824

Morrison, S. J., and Scadden, D. T. (2014). The bone marrow niche for haematopoietic stem cells. Nature 505, 327-334. doi: 10.1038/nature12984

Murke, F., Castro, S. V. C., Giebel, B., and Görgens, A. (2015). Concise review: asymmetric cell divisions in stem cell biology. Symmetry 7, 2025-2037. doi: $10.3390 /$ sym7042025

Oritani, K., Aoyama, K., Tomiyama, Y., Kincade, P. W., and Matsuzawa, Y. (2000). Stromal cell CD9 and the differentiation of hematopoietic stem/progenitor cells. Leuk Lymphom. 38, 147-152. doi: 10.3109/10428190009060328

Papayannopoulou, T., Craddock, C., Nakamoto, B., Priestley, G. V., and Wolf, N. S. (1995). The VLA4/VCAM-1 adhesion pathway defines contrasting mechanisms of lodgement of transplanted murine hemopoietic progenitors between bone marrow and spleen. Proc. Natl. Acad. Sci. U.S.A. 92, 9647-9651. doi: 10.1073/ pnas.92.21.9647

Peled, A., Kollet, O., Ponomaryov, T., Petit, I., Franitza, S., Grabovsky, V., et al. (2000). The chemokine SDF-1 activates the integrins LFA-1, VLA-4, and VLA-5 on immature human CD34(+) cells: role in transendothelial/stromal migration and engraftment of NOD/SCID mice. Blood 95, 3289-3296. doi: 10.1182/blood. v95.11.3289.011k33_3289_3296

Pinho, S., and Frenette, P. S. (2019). Haematopoietic stem cell activity and interactions with the niche. Nat. Rev. Mol. Cell Biol. 20, 303-320. doi: 10.1038/ s41580-019-0103-9
Qian, H., Tryggvason, K., Jacobsen, S. E., and Ekblom, M. (2006). Contribution of alpha6 integrins to hematopoietic stem and progenitor cell homing to bone marrow and collaboration with alpha4 integrins. Blood 107, 3503-3510. doi: 10.1182/blood-2005-10-3932

Reyes, R., Cardenes, B., Machado-Pineda, Y., and Cabanas, C. (2018). Tetraspanin CD9: a key regulator of cell adhesion in the immune system. Front. Immunol. 9:863. doi: 10.3389/fimmu.2018.00863

Reyes, R., Monjas, A., Yanez-Mo, M., Cardenes, B., Morlino, G., Gilsanz, A., et al. (2015). Different states of integrin LFA-1 aggregation are controlled through its association with tetraspanin CD9. Biochim. Biophys. Acta 1853(10 Pt A), 2464-2480. doi: 10.1016/j.bbamcr.2015.05.018

Robb, L., Tarrant, J., Groom, J., Ibrahim, M., Li, R., Borobakas, B., et al. (2001). Molecular characterisation of mouse and human TSSC6: evidence that TSSC6 is a genuine member of the tetraspanin superfamily and is expressed specifically in haematopoietic organs. Biochim. Biophys. Acta 1522, 31-41. doi: 10.1016/ s0167-4781(01)00306-2

Rossi, L., Forte, D., Migliardi, G., Salvestrini, V., Buzzi, M., Ricciardi, M. R., et al. (2015). The tissue inhibitor of metalloproteinases 1 increases the clonogenic efficiency of human hematopoietic progenitor cells through CD63/PI3K/Akt signaling. Exp. Hematol. 43, 974-985. doi: 10.1016/j.exphem.2015.07.003

Saito-Reis, C. A., Marjon, K. D., Pascetti, E. M., Floren, M., and Gillette, J. M. (2018). The tetraspanin CD82 regulates bone marrow homing and engraftment of hematopoietic stem and progenitor cells. Mol. Biol. Cell 29, 2946-2958. doi: 10.1091/mbc.E18-05-0305

Schofield, R. (1978). The relationship between the spleen colony-forming cell and the haemopoietic stem cell. Blood Cells 4, 7-25.

Schwartz-Albiez, R., Dorken, B., Hofmann, W., and Moldenhauer, G. (1988). The B cell-associated CD37 antigen (gp40-52). Structure and subcellular expression of an extensively glycosylated glycoprotein. J. Immunol. 140, 905-914.

Spring, F. A., Griffiths, R. E., Mankelow, T. J., Agnew, C., Parsons, S. F., Chasis, J. A., et al. (2013). Tetraspanins CD81 and CD82 facilitate alpha4betal-mediated adhesion of human erythroblasts to vascular cell adhesion molecule-1. PLoS One 8:e62654. doi: 10.1371/journal.pone.0062654

Stik, G., Crequit, S., Petit, L., Durant, J., Charbord, P., Jaffredo, T., et al. (2017). Extracellular vesicles of stromal origin target and support hematopoietic stem and progenitor cells. J. Cell Biol. 216, 2217-2230. doi: 10.1083/jcb.201601109

Stipp, C. S., Kolesnikova, T. V., and Hemler, M. E. (2003). Functional domains in tetraspanin proteins. Trends Biochem. Sci. 28, 106-112. doi: 10.1016/S09680004(02)00014-2

Szade, K., Gulati, G. S., Chan, C. K. F., Kao, K. S., Miyanishi, M., Marjon, K. D., et al. (2018). Where hematopoietic stem cells live: the bone marrow niche. Antioxid. Redox Signal. 29, 191-204. doi: 10.1089/ars.2017.7419

Tai, X. G., Yashiro, Y., Abe, R., Toyooka, K., Wood, C. R., Morris, J., et al. (1996). A role for CD9 molecules in T cell activation. J. Exp. Med. 184, 753-758. doi: $10.1084 /$ jem.184.2.753

Tedder, T. F., Zhou, L. J., and Engel, P. (1994). The CD19/CD21 signal transduction complex of B lymphocytes. Immunol. Today 15, 437-442. doi: 10.1016/01675699(94)90274-7

Termini, C. M., Cotter, M. L., Marjon, K. D., Buranda, T., Lidke, K. A., and Gillette, J. M. (2014). The membrane scaffold CD82 regulates cell adhesion by altering alpha4 integrin stability and molecular density. Mol. Biol. Cell 25, 1560-1573. doi: 10.1091/mbc.E13-11-0660

Termini, C. M., and Gillette, J. M. (2017). Tetraspanins function as regulators of cellular signaling. Front. Cell Dev. Biol. 5:34. doi: 10.3389/fcell.2017.00034

Todres, E., Nardi, J. B., and Robertson, H. M. (2000). The tetraspanin superfamily in insects. Insect. Mol. Biol. 9, 581-590. doi: 10.1046/j.1365-2583.2000.00222.x

Umeda, R., Satouh, Y., Takemoto, M., Nakada-Nakura, Y., Liu, K., Yokoyama, T., et al. (2020). Structural insights into tetraspanin CD9 function. Nat. Commun. 11:1606. doi: 10.1038/s41467-020-15459-7

van Deventer, S. J., Dunlock, V. E., and van Spriel, A. B. (2017). Molecular interactions shaping the tetraspanin web. Biochem. Soc. Trans. 45, 741-750. doi: 10.1042/BST20160284

van Zelm, M. C., Smet, J., Adams, B., Mascart, F., Schandene, L., Janssen, F., et al. (2010). CD81 gene defect in humans disrupts CD19 complex formation and leads to antibody deficiency. J. Clin. Invest. 120, 1265-1274. doi: 10.1172/ JCI39748

Weiss-Gayet, M., Starck, J., Chaabouni, A., Chazaud, B., and Morle, F. (2016). Notch stimulates both self-renewal and lineage plasticity in a subset of murine 
CD9High committed megakaryocytic progenitors. PLoS One 11:e0153860. doi: 10.1371/journal.pone.0153860

Wilk, C. M., Schildberg, F. A., Lauterbach, M. A., Cadeddu, R. P., Frobel, J., Westphal, V., et al. (2013). The tissue inhibitor of metalloproteinases-1 improves migration and adhesion of hematopoietic stem and progenitor cells. Exp. Hematol. 41, 823-831. doi: 10.1016/j.exphem.2013.04.010

Wilson, A., Laurenti, E., Oser, G., van der Wath, R. C., Blanco-Bose, W., Jaworski, M., et al. (2008). Hematopoietic stem cells reversibly switch from dormancy to self-renewal during homeostasis and repair. Cell 135, 1118-1129. doi: 10.1016/ j.cell.2008.10.048

Yunta, M., and Lazo, P. A. (2003). Tetraspanin proteins as organisers of membrane microdomains and signalling complexes. Cell Signal. 15, 559-564.

Zhang, J., Niu, C., Ye, L., Huang, H., He, X., Tong, W. G., et al. (2003). Identification of the haematopoietic stem cell niche and control of the niche size. Nature 425, 836-841. doi: 10.1038/nature02041

Zhao, Y., Wang, H., and Mazzone, T. (2006). Identification of stem cells from human umbilical cord blood with embryonic and hematopoietic characteristics. Exp. Cell Res. 312, 2454-2464. doi: 10.1016/j.yexcr.2006.04.008
Zimmerman, B., Kelly, B., McMillan, B. J., Seegar, T. C. M., Dror, R. O., Kruse, A. C., et al. (2016). Crystal structure of a full-length human tetraspanin reveals a cholesterol-binding pocket. Cell 167, 1041-1051. doi: 10.1016/j.cell.2016.09.056 Zou, F., Wang, X., Han, X., Rothschild, G., Zheng, S. G., Basu, U., et al. (2018). Expression and function of tetraspanins and their interacting partners in B cells. Front. Immunol. 9:1606. doi: 10.3389/fimmu.2018.01606

Conflict of Interest: The authors declare that the research was conducted in the absence of any commercial or financial relationships that could be construed as a potential conflict of interest.

Copyright (c) 2020 Balise, Saito-Reis and Gillette. This is an open-access article distributed under the terms of the Creative Commons Attribution License (CC BY). The use, distribution or reproduction in other forums is permitted, provided the original author(s) and the copyright owner(s) are credited and that the original publication in this journal is cited, in accordance with accepted academic practice. No use, distribution or reproduction is permitted which does not comply with these terms. 